\title{
CMB: ANISOTROPIES DUE TO NON-LINEAR CLUSTERING
}

\author{
E. MARTíNEZ-GONZÁLEZ AND J. L. SANZ \\ Dpto. de Física Moderna, Universidad de Cantabria and \\ Instituto de Estudios Avanzados, \\ CSIC-Universidad de Cantabria \\ Facultad de Ciencias, Avda. de Los Castros s/n, \\ 39005 Santander, Spain
}

Most of the studies on the anisotropy expected in the temperature of the cosmic microwave background (CMB) have been based on linear density perturbations. The anisotropies at angular scales $\geq 1^{\circ}$ (horizon at recombination) are preserved during the evolution of the universe, whereas for smaller scales new effects can appear, generated during the non-linear phase of matter clustering evolution: i) the Sunyaev-Zeldovich effect due to hot gas in clusters (Scaramella et al. 1993), ii) the Vishniac effect (Vishniac 1987) due to the coupling between density fluctuations and bulk motions of gas and iii) the integrated gravitational effect (Martínez-González et al. 1994) due to time-varyng gravitational potentials. A single potential $\phi(t, \mathbf{x})$, satisfying the Poisson equation, is enouph to describe weak gravitational fields associated to non-linear density fluctuations when one considers scales smaller than the horizon and non-relativistic peculiar velocities. The temperature anisotropies, in a flat universe, are given by the expression (Martínez-González et al. 1990)

$$
\frac{\Delta T}{T}=\frac{1}{3} \phi_{r}+\mathbf{n} \cdot \mathbf{v}_{\mathbf{r}}+2 \int_{r}^{o} d t \frac{\partial \phi}{\partial t}(t, \mathbf{x}),
$$

where $\mathbf{n}$ is the unit vector in the direction of observation, the subscript $r$ denotes the recombination time and we have chosen units such that $8 \pi G=c=1$. The temperature correlation function $C(\alpha)$ can be obtained from the previous equation by averaging over all direction pairs separated by an angle $\alpha$ and it is given by the following integrated effect

$$
C(\alpha)=4 \int_{0}^{1} d \lambda_{1} \int_{0}^{1} d \lambda_{2} \frac{\partial^{2}}{\partial \lambda_{1} \partial \lambda_{2}} C_{\phi}\left(\lambda_{1}, \lambda_{2} ; x\right),
$$


where $x^{2}=\lambda_{1}^{2}+\lambda_{2}^{2}-2 \lambda_{1} \lambda_{2} \cos \alpha, \lambda_{1}$ and $\lambda_{2}$ represent the distances to the two photons from the observer and $C_{\phi}$ is the correlation of the gravitational potential. As a model for the evolution of the correlation of matter $\xi\left(z_{1}, z_{2} ; r\right)$, we will assume

$$
\xi\left(z_{1}, z_{2} ; r\right)=\left[\left(1+z_{1}\right)\left(1+z_{2}\right)\right]^{-\frac{3+\epsilon}{2}}\left(\frac{r_{0}}{r}\right)^{\gamma}, \quad r \leq r_{m},
$$

and $\xi=0$ otherwise. The values we will consider are $\gamma \simeq 1.8$ and $r_{o} \simeq 5 h^{-1} M p c$ based on observations of the correlation function of galaxies at the present epoch. The evolution in redshift is parametrized in terms of $\epsilon$, the value $\epsilon=0,-1.2$ represent stable and comoving clustering, respectively.

The main results we have obtained can be summarized as follows: i) the temperature correlation function $10^{-6} \leq C(\alpha)^{1 / 2} \leq 10^{-5}$ for values of the evolution parameter $-1.2 \leq \epsilon \leq 3$, initial redshift $z_{m} \geq 3$ and cut-off $r_{m} \geq 10 h^{-1} M p c$ at present. ii) $\Delta T / T$ is maximum for comoving clustering $(\epsilon=-1.2)$, decreases for stable clustering $(\epsilon=0)$ and slowly increases for $\epsilon \geq 0$. iii) More than $80 \%$ of the effect is produced in the interval $z \leq 3$ for $\epsilon \geq 0$, whereas for comoving clustering the effect is created near the initial redshift. iv) Considering present experimental limits on degree scales $\left(\Delta T / T \leq 10^{-5}\right)$, the non-linear evolution of the correlation function for comoving clustering must have started at $z \leq 10$ if $r_{m}=10 h^{-1} M p c$. Extrapolating to $r_{m}=19 h^{-1} M p c$, those limits imply that clusters must have formed by $z \leq 4$ for the same type of evolution. $\mathrm{v}$ ) The stable clustering model or models with a faster evolution $(\epsilon>0)$ give $\Delta T / T$ of the order of $10^{-6}$, so they do not violate present upper-limits.

\section{References}

Martínez-González, E., Sanz, J.L. and Silk, J.(1990), The Astrophysical Journal Letters, Vol. no. 355, L5.

Martínez-González, E., Sanz, J.L. and Silk, J.(1994), The Astrophysical Journal, in press.

Scaramella, R., Cen, R. and Ostriker, J. P. (1993), The Astrophysical Journal, Vol. no. 416, 399.

Vishniac, E. T. (1987), The Astrophysical Journal,Vol. no. 322 ,597. 